\title{
Molecular diagnosis of invasive aspergillosis in a child with clinical suspicion of mucormycosis
}

\author{
R Sabino', A Ramos ${ }^{2}$, H Simões ${ }^{1}$, MJ Palaré ${ }^{3}$, R Moreno4, A Ferrão3, \\ F Lourenço ${ }^{4}$, JG Marques ${ }^{5}$, C Martins ${ }^{4}$, A Morais ${ }^{3}$, C Verissimo ${ }^{1}$
}

\begin{abstract}
1- Reference Unit for Parasitic and fungal Infections, Infectious Diseases Department, National Institute of Health Dr. Ricardo Jorge Lisbon, Portugal 2-Internship with specialization in Clinical Hematology, Hematology and Bone Marrow Transplant Ward, North Lisbon Hospital Centre, EPE, Lisbon, Portugal

3- Pediatrics Hematology Unit - Clinical Pediatrics Ward, North Lisbon Hospital Centre, EPE, Lisbon, Portugal

4- Bone Marrow Transplant Unit - Hematology and Bone Marrow Transplant Ward, North Lisbon Hospital Centre, EPE, Lisbon, Portugal

5- Pediatrics Infecciology Unit - Clinical Pediatrics Ward, North Lisbon Hospital Centre, EPE, Lisbon, Portugal
\end{abstract}

\section{Objectives:}

Clinical case of a 13-year-old boy from Cabo Verde who was transferred to the Hematology Unit of a Central Hospital in Lisbon, Portugal, and diagnosed with aplastic anemia. Weeks after hospital admission, in June 2016, he presented febrile neutropenia with negative blood cultures. In August, the patient showed a necrotic lesion of the left wing of the nose (Fig. 1). A CT scan of the sinuses showed densification and thickening of the soft tissues of the nasal pyramid, protruding into the nose. A rapid and accurate diagnosis was required since the patient was going to be subjected to bone marrow transplantation.

\section{Methods:}

- A polyphasic approach was performed in order to detect and identify the etiological fungal agent in the analysed tissue sample (Fig. 2):

- Tissue fragments biopsy of the nose were sliced in small fragments and inoculated in Sabouraud dextrose agar and brain heart infusion media. Samples were incubated at $30^{\circ}$ and $35^{\circ} \mathrm{C}$ (Fig. 3).

- In parallel to culture, DNA was extracted from the tissue using the High Pure PCR Template Preparation Kit (Roche Diagnostics Corp., Indianapolis, IN, USA), according to the manufacturer's instructions.

- A panfungal PCR reaction was performed in order to detect any fungal DNA present in the tissue sample. For that purpose, the universal fungal primers ITS1 and ITS2 were used. Positive PCR products are then sequenced by Sanger method.

- A specific PCR directed to Aspergillus was performed directly from the DNA extracted from the biopsy using the AsperGenius ${ }^{\circledR}$ multiplex real-time PCR assay (PathoNostics, Maastricht, The Netherlands), following the manufacturer's instructions.

-The detection of mutations in the Cyp51A gene for $A$. fumigatus conferring azole resistance was also performed.

\section{Results:}

A positive panfungal PCR was obtained (Fig. 4) and PCR products were sent to sequencing. Sequencing results revealed $100 \%$ homology with $A$. fumigatus sensu stricto.

The real time PCR of the sample obtained positive signal for Aspergillus fumigatus and no mutations in Cyp51A gene were detected (Fig. 5).

Cultures remained negative after more than 30 days of incubation.

Posaconazole was administered with regression of the lesion. In September 2016, nodular lesions appeared in the patients' lower limbs and voriconazole therapy was then initiated. A complete regression was observed.

Multiresistant Klebsiella pneumoniae and Enterobacter asburiae were further isolated from a perianal ulcer and in October 2016 the patient revealed positive bloodcultures of multiresistant Klebsiella pneumoniae.

Facing difficulties in the infection control, a multidisciplinary team decided to perform allogeneic bone marrow transplantation, with complete hematological and infections recovery.

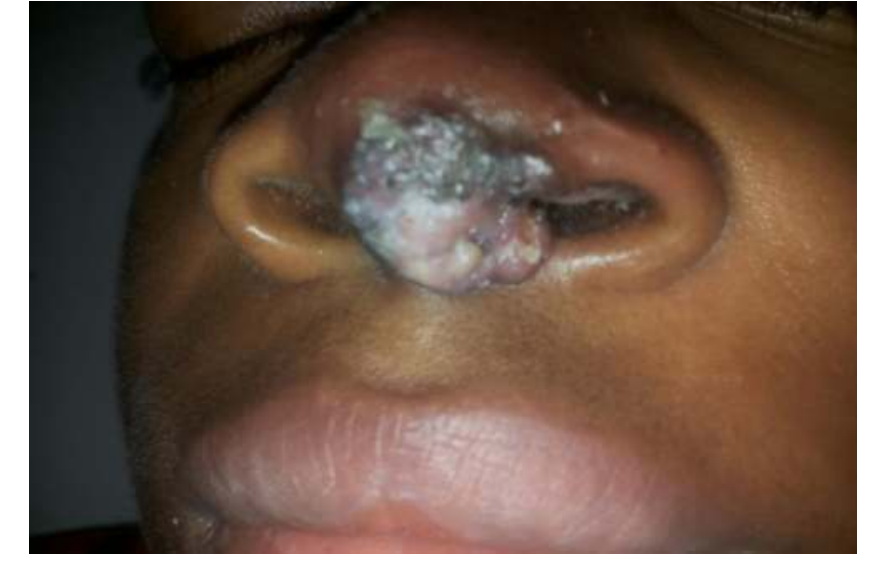

Fig.1. Nasal lesion of the patient

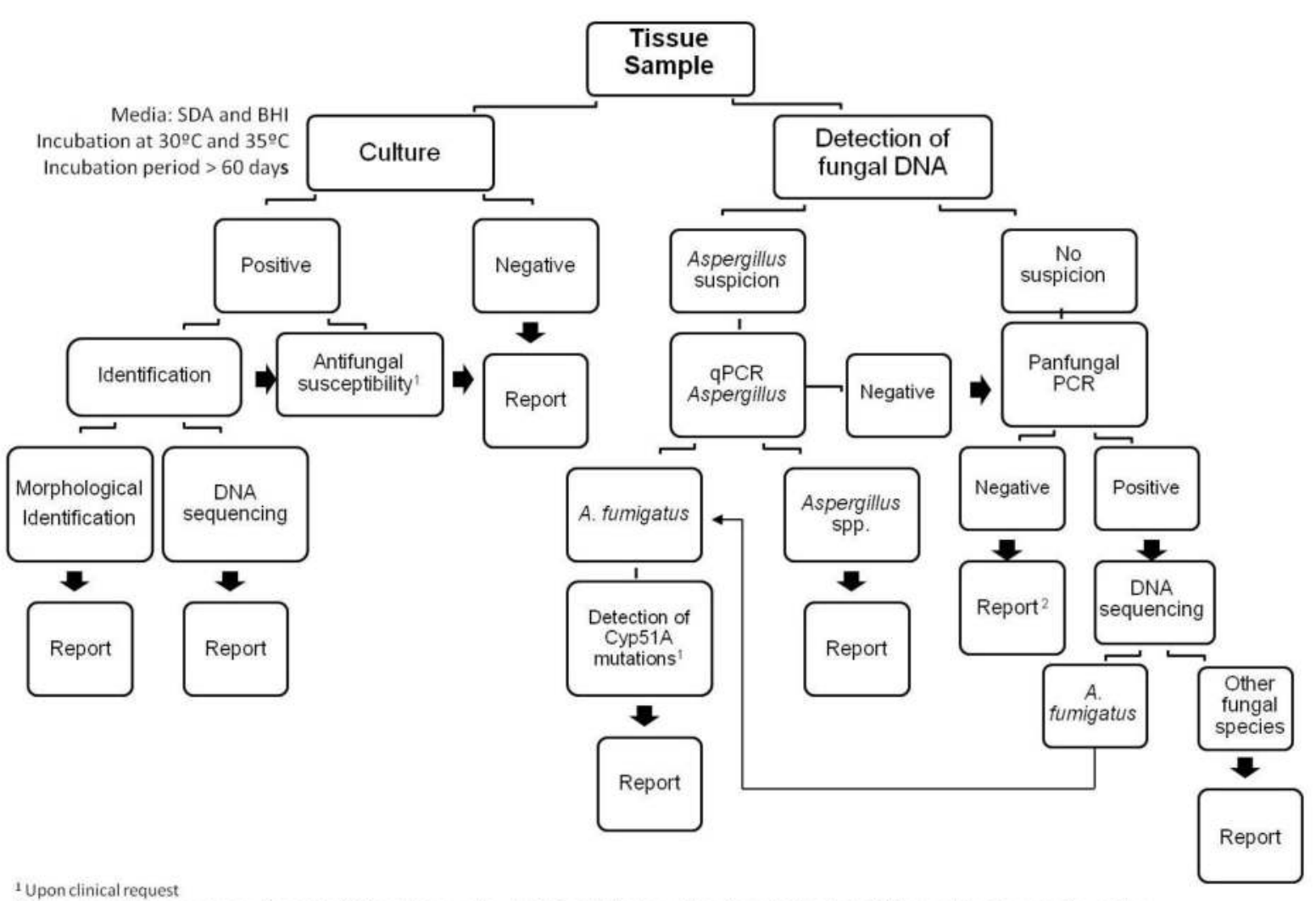

Fig. 2. Polyphasic diagnostic approach for the detection and identification of fungi in tissue samples

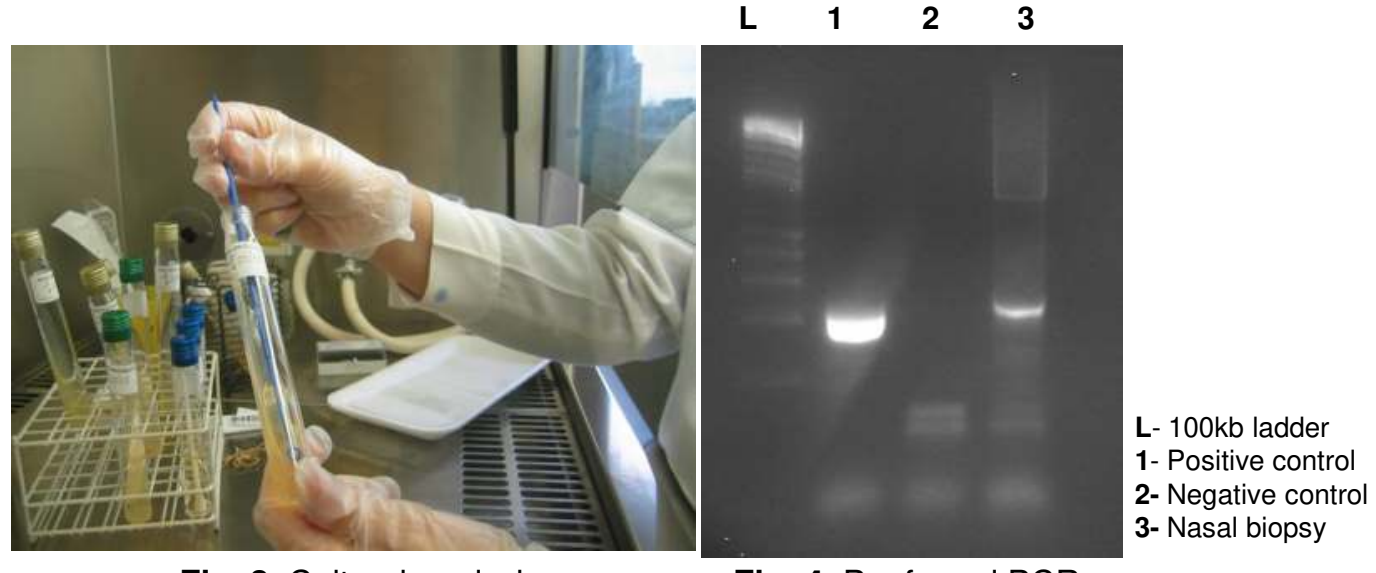

Fig. 3. Cultural analysis $\quad$ Fig. 4. Panfungal PCR
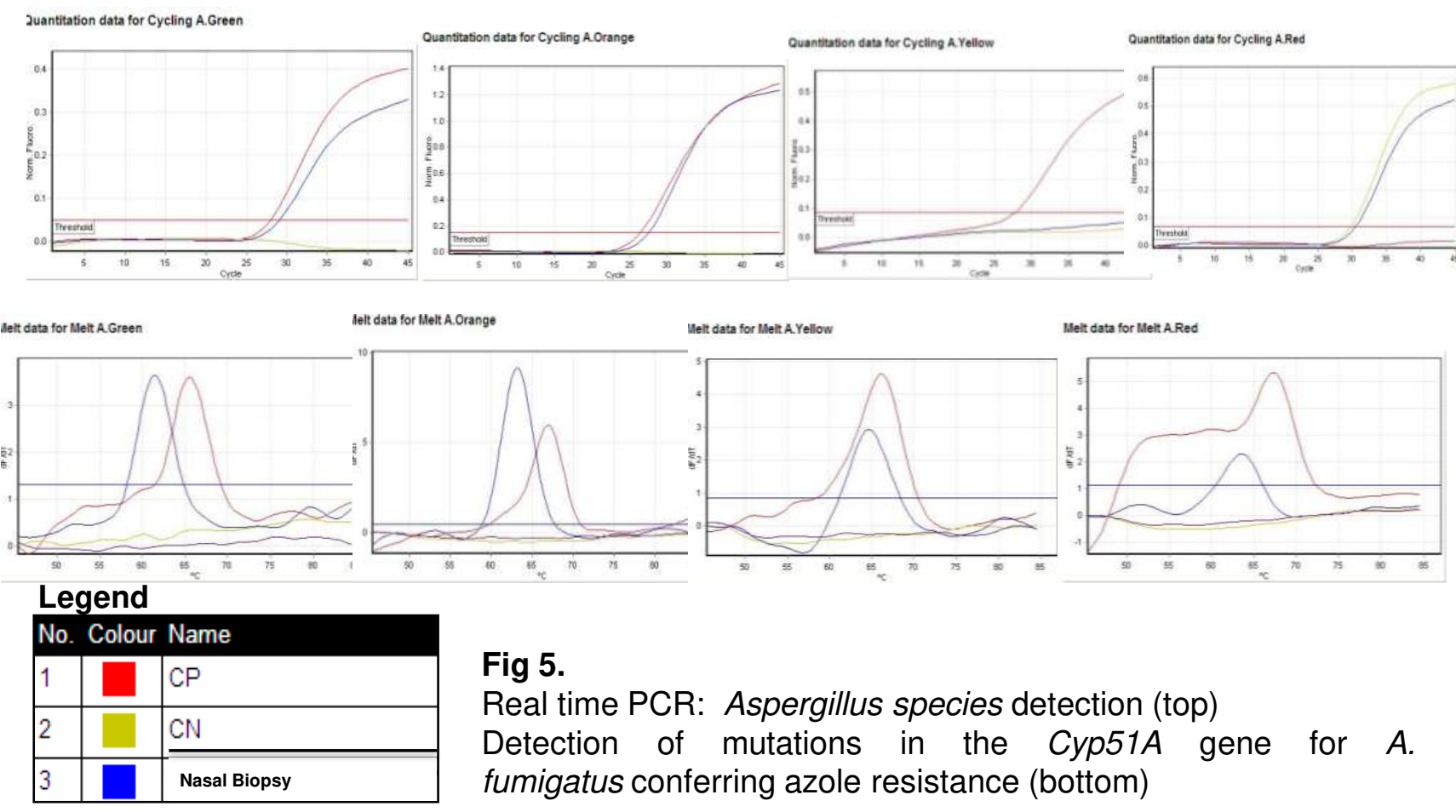

Conclusion:

An early diagnosis and prompt initiation of appropriate antifungal therapy are imperative and essential for a favorable clinical outcome. In this case, the necrotic lesions of the nose and patient's risk factors conducted to the clinical suspicion of mucormycosis. The molecular approach performed led to the rapid identification of Aspergillus fumigatus and therefore to the adequate antifungal therapy of the patient. 